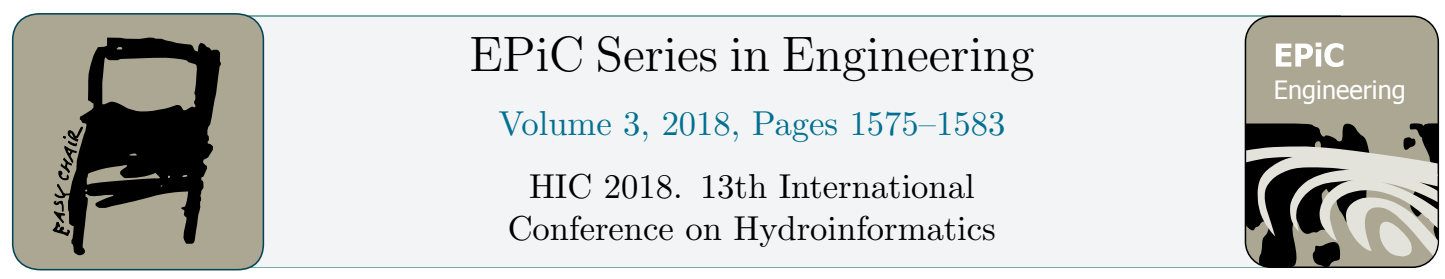

\title{
QGIS FLO-2D Integration
}

\author{
Karen O’Brien, BSc. ${ }^{1}$, Noemi Gonzalez-Ramirez, Ph. D. ${ }^{1}$ and Fernando \\ Nardi, Ph. D. ${ }^{2}$ \\ 1 FLO-2D Software, Inc., P.O. Box 66, Nutrioso, AZ 85932, United States \\ 2 Università per Stranieri di Perugia, Piazza Fortebraccio, 4. 06123, Perugia Area, Italy. \\ karen@flo-2d.com, noemi@flo-2d.com, fernando.nardi@unistrapg.it
}

\begin{abstract}
Cities and agencies worldwide are predicting complex urban flooding by simulating the exchange between the surface water and storm drain system with the FLO-2D model. The FLO-2D modeling system is now supported by the robust pre- and post-processor tools in the Quantum Graphical Information Systems (QGIS) geographic information software. The QGIS tool prepares the FLO-2D data base that includes analyzing and editing spatial information as well as exporting detailed and high-resolution flood hazard maps. This paper outlines the resources and modeling tools available to engineers in an open source and free cross platform geographic information tool that supports the viewing, creation and editing of the spatial and tabular data as well as graphical and mapping output. This paper discusses the features of this unique, free, efficient and comprehensive data processing tool. A case study for the application of the QGIS tool for a FLO-2D urban project is presented.
\end{abstract}

\section{Overview}

The tools for developing large scale two-dimensional flood models have expanded in the past decade to enable engineers to tackle virtually every detail of the complex urban environment. These tools can now generate the data base to represent surface and subsurface features to capture in high resolution the physical processes of floodwave movement. The ability to manage and manipulate mega data sets is essential for today's flood studies. Urban data sets are typically large and ultimately need to be refined to the resolution of individual buildings. The data base tools and spatial mapping must be as robust and efficient as the flood routing engines. In terms of flood hazard and risk, it is no longer feasible for a flood study to be developed for a single use. Graphical Information System (GIS) software, modern data storage servers and highly refined flood routing engines provide flood management agencies with the capability to create and maintain models that evolve as their municipalities develop. This paper will discuss the QGIS plugin and the Geospatial Data Management System components that were developed within the FLO-2D system to accommodate the tool. 


\section{Quantum Geographic Information System}

QGIS is an open source geographical information system that is free to all users. It relies on a network of developers around the globe to create tools and plugins that are found in most GIS programs. Various GIS data formats can be imported into or created by QGIS. This includes raster, vector data, vector attributes, delimited text, and topology. It is a 64-Bit program that can use multiple processors and multiple threads to manipulate and develop spatial and tabular mega data sets (QGIS, 2018). The data is packaged and arranged so that the entire project dataset is readily accessible.

QGIS has a comprehensive collection of Geospatial Data Abstraction Library (GDAL) tools. These are tools used to read, write and manipulate raster and vector data. The tools are both built into the software and loaded as extra plugins that perform specific commands such as point samples and raster alignment.

The program window is arranged into various viewing and editing panels (Figure 1). These include many toolbars, layer and identification panels, a main map area and extras like dockable plotting windows and tables.

Each project loaded into QGIS can be arranged by layers and grouped. Comprehensive styling options are available to help end users create map data that facilitate data comparisons. The layering and styles, coordinate reference systems and layer data locations are compiled into a project file so that each time the project is opened, all view and data configurations are maintained.

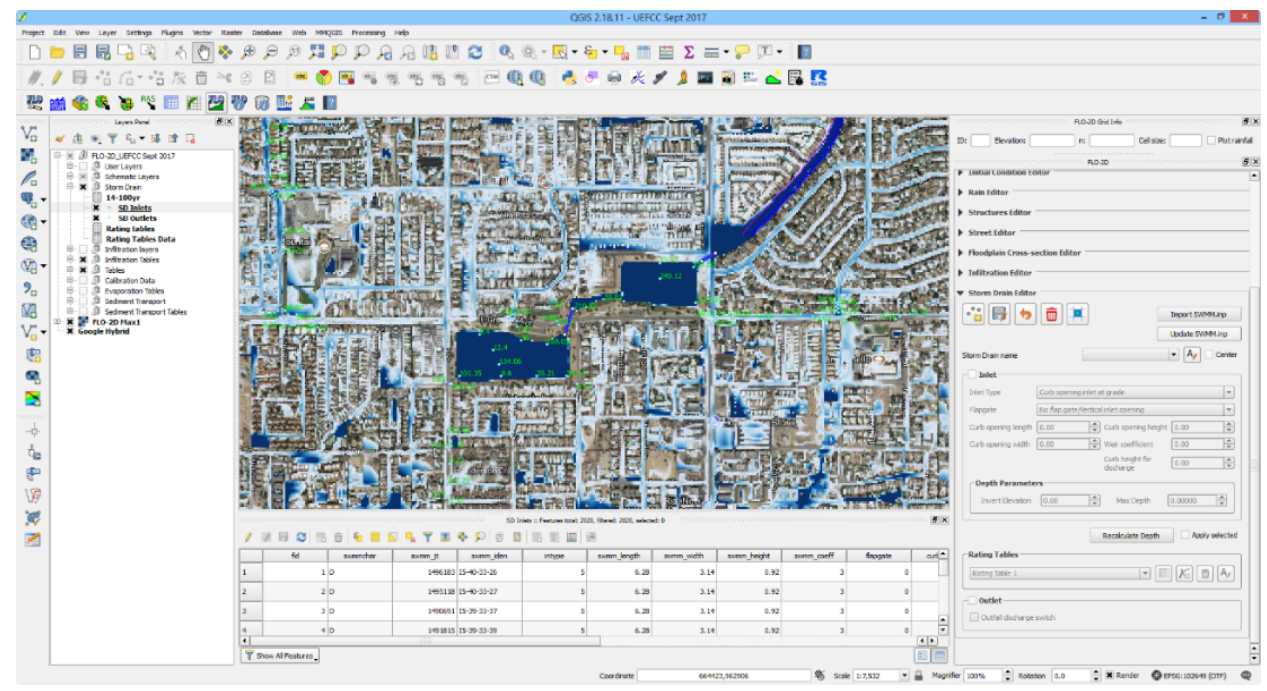

Figure 1: QGIS Project Layout

\section{Data Management Systems}

Urban flood studies include data from a wide range of resources. A major challenge for agencies and municipalities is data management. Projects may have mapping or data resources that contain elements of digital elevation, hydrology and hydraulics, design drawings, aerial images, storm data, tax data, environmental data and field study notes and pictures (Figure 2). Mapping software such as QGIS and ESRI ArcMap have online interface tools so projects can be stored online or on data servers that are controlled by the agency or a consulting team. 


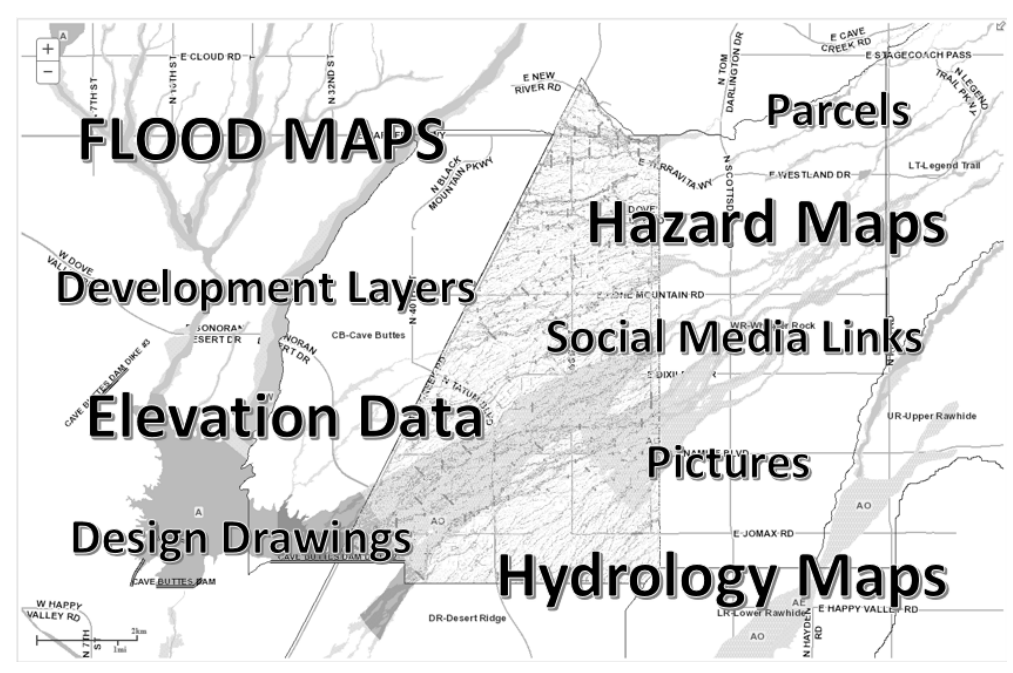

Figure 2: Complex Data Management

Web mapping tools (Figure 3) can be linked to municipality and agency websites and stored on their servers. These tools can be shared with interested parties at various levels of security. The general public can have basic access to flood maps, hazard and safety maps, tax maps and aerial images, and social media links. Engineers and consultants can access a larger dataset that includes design data, hydraulic results, hydrology data, and elevation data. Engineers or mapping personnel can create hyperlinks to field data or known flood prone areas. The hyperlinks are pinned directly to the map. Once the user clicks the link, data is presented in different formats. The link may open a table of results, a field image, a video or even a design drawing. The final layer of security rests with the IT department. They are responsible for maintaining the repository of all data and preventing data loss. This includes all project data sets, all supporting map data, all documentation, and flood control procedural policies. 

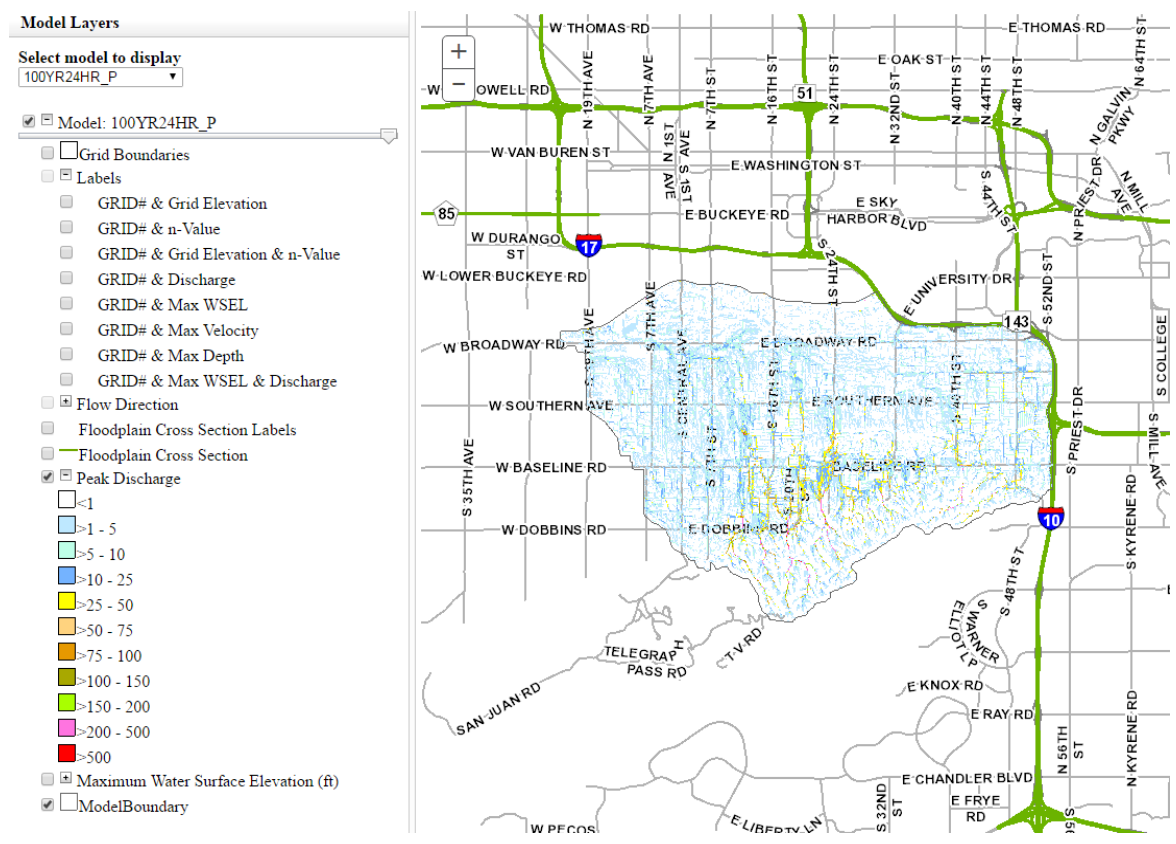

Figure 3: Flood Control District of Maricopa County, Arizona Web Access Tool

\section{FLO-2D Model QGIS Plugin}

The FLO-2D model is a fully integrated 2-D surface flooding and storm drain model combining hydrology and flood hydraulics (O' Brien, 2012) (FLO-2D, 2018). It is specifically developed for use on urban flood studies (Figure 4). The urban model components include spatially and temporally variable rainfall, infiltration, channel and street flow, gutter flow, hydraulic structures, storm drain system, building loss of storage and flow obstruction, building roof runoff, building collapse, berms, levees and walls and wall collapse, groundwater flow, mudflow and sediment transport, and low impact development (LID). 


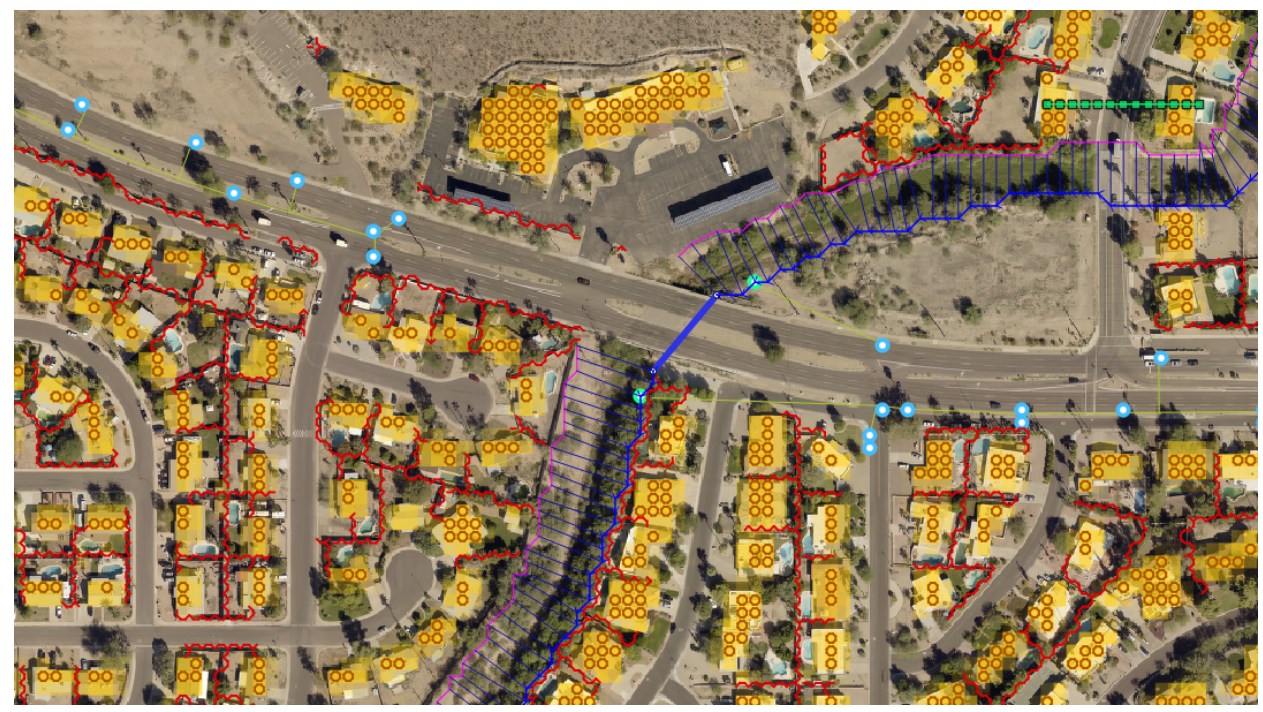

Figure 4: FLO-2D complex urban model

A plugin was developed for QGIS to support the creation, editing and visualization of the FLO-2D data. The plugin is a free cross platform geographic information tool that supports the viewing, creation and editing of the FLO-2D component data and allows the user to explore all the spatial information capabilities including both pre- and post-processing mapping and images.

The QGIS plugin supports raster and vector layers and creates the FLO-2D model files. It can be used to digitize data for the FLO-2D model. The plugin can import project data, create data used by the FLO-2D Model and export the FLO-2D data files. Some of the QGIS plugin attributes are discussed below.

\subsection{Editing tool bar}

The plugin package includes a toolbar for building the project geographical system and has icons for editing the various FLO-2D component data files. Figure 5 represents a list of the currently available menu commands for the basic FLO-2D model data file structure. The QGIS plugin has all the editor functions of the GDS and additional tools are available.

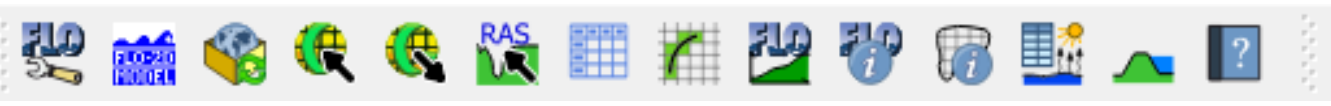

Figure 5: FLO-2D Toolbar

\subsection{Spatially variable surface editors}

The grid tools are used to compute spatially variable data to the surface layer and grid layer of a FLO-2D Project (Figure 6).

- Computational domain and grid

- Sample and interpolate elevation data, breaklines, polygon elevation corrections

- Roughness 
- Buildings

- Low impact development (LID)

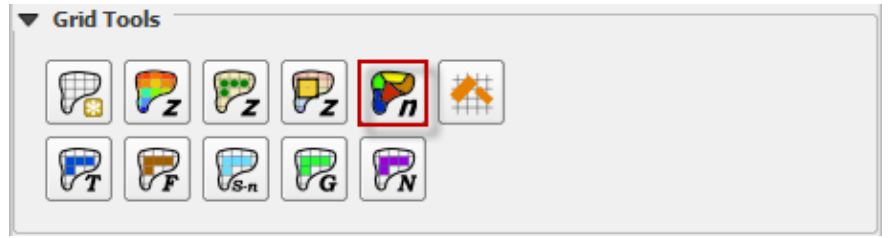

Figure 6: Grid Editor Tools

\subsection{Linear 1-D feature editors}

Inline features such as channels, streets, levees and hydraulic structures can be edited with a combinations of polyline vectors and points to help place data features such as channel geometry, culvert and bridge locations, gutters and street breaklines and levee lines and crests. Figure 7 shows an example of a natural channel and low flow culvert.

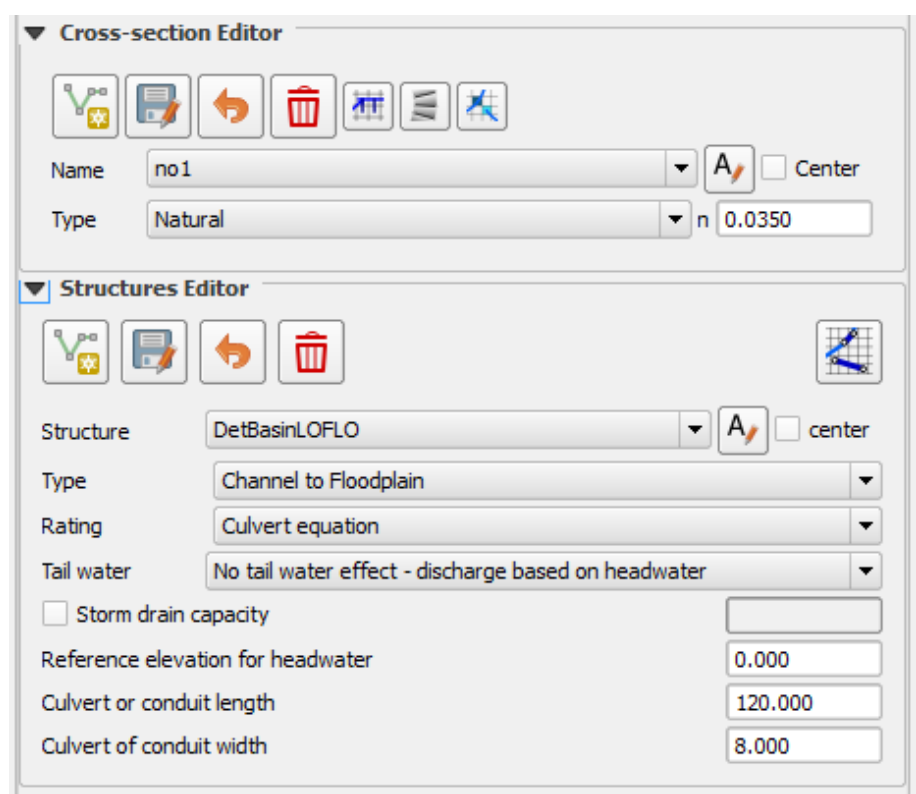

Figure 7: 1-D Channel Editor Dialog Box

\subsection{Hydrology editor}

FLO-2D is a combined hydrologic and hydraulic model. The plugin editors can define data for several types of rainfall and infiltration conditions (Figure 8). The tool can interpolate real time spatially and temporally variable rainfall data, uniform rainfall data and depth area reduction data. Infiltration tools included a Green-Ampt calculator that uses Land Use and Soils shapefiles to define the spatially variable infiltration parameters. 


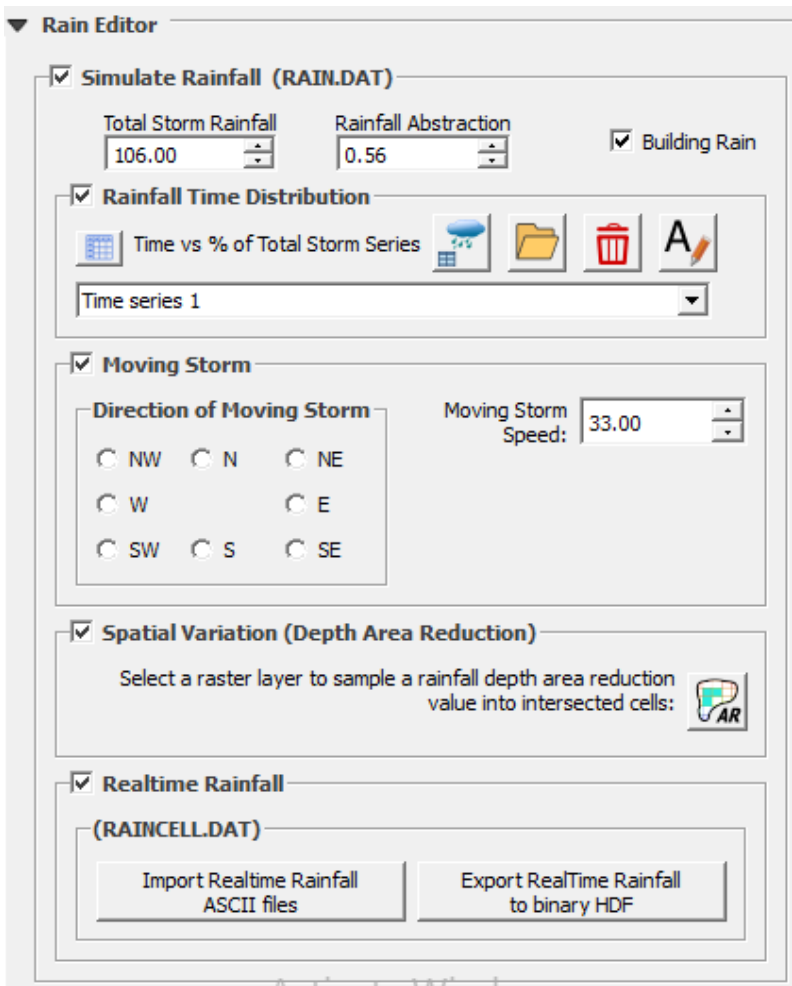

Figure 8: Rainfall Editor

\subsection{Storm Drain Editor}

FLO-2D can simulate the flow exchange between the surface and the storm drain system. The storm drain editor (Figure 9) is used to digitize, import or load storm drain network data and append it to the FLO-2D grid system and channels. The import tool (Figure 10) is used to import a complete storm drain network from inlet, outfall and conduit shapefiles.

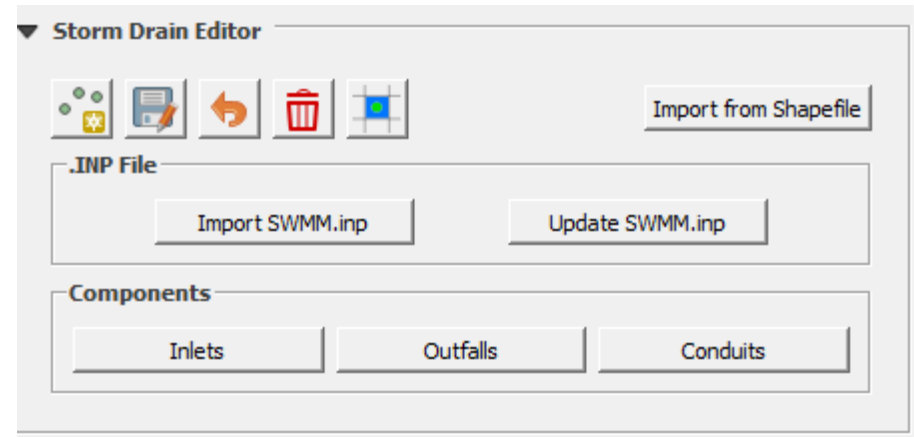

Figure 9: Storm Editor 


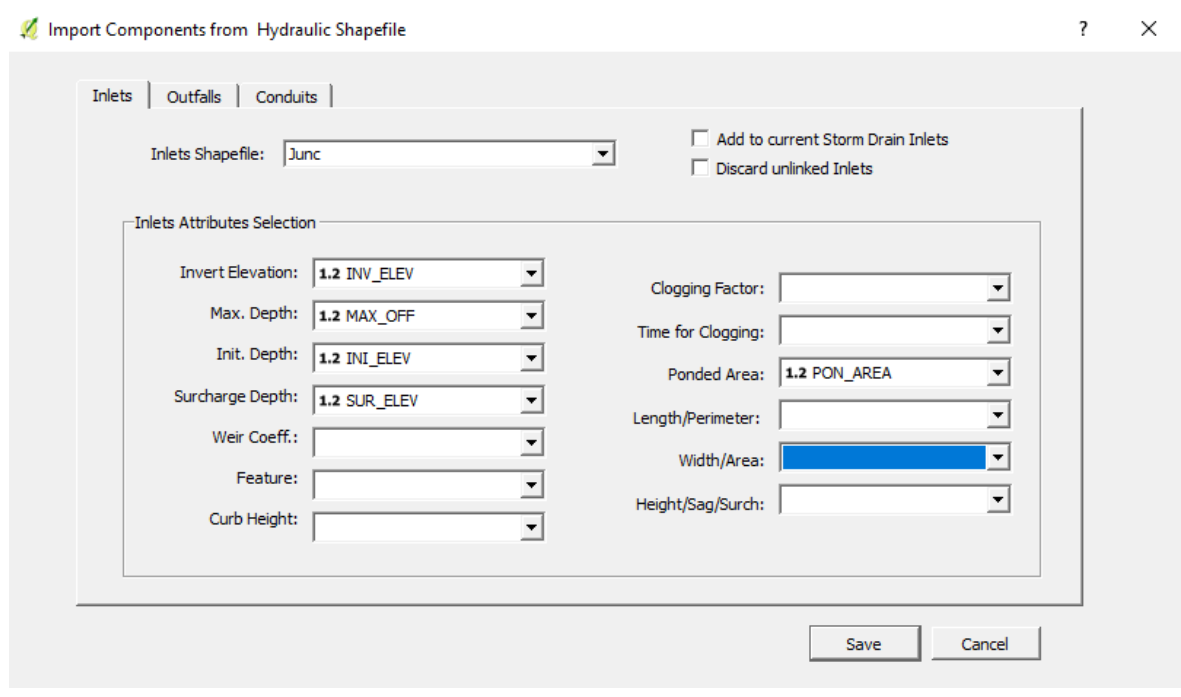

Figure 10: Import Storm Drain Features from Shapefiles

\section{Comprehensive Urban Modelling}

Facilitating data input through a GIS based GUI is critical for a large urban model. A recent storm drain model for a suburb of Phoenix, Arizona had 1,855,137 $20 \mathrm{ft}(6.1 \mathrm{~m})$ grid elements, three channels, six detention basins, hundreds of walls and embankments, thousands of buildings and an extensive storm drain system that included 2,700 closed conduits $(313,000 \mathrm{ft}-95,400 \mathrm{~m}), 2,030$ inlets and 97 outfalls (Figure 11). The $24 \mathrm{hr}$ storm simulation model took approximately $12 \mathrm{hrs} \mathrm{runtime.}$

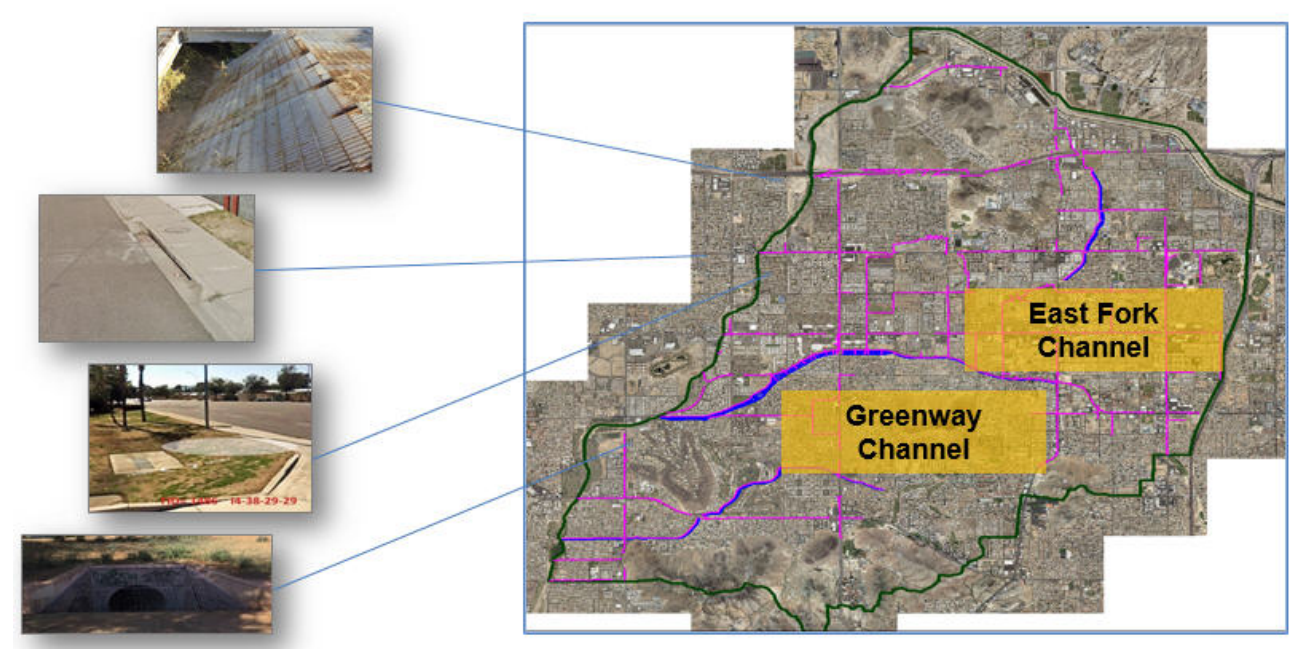

Figure 11: Extensive FLO-2D Urban Surface Water and Storm Drain Model for Phoenix, Arizona 
To visualize the model results requires a high-resolution GIS mapping tool. Flood maps can be plotted as shapefiles, rasters, points, vectors or contours. QGIS can read and plot the flood maps in any format. The mapping can be edited or exported.

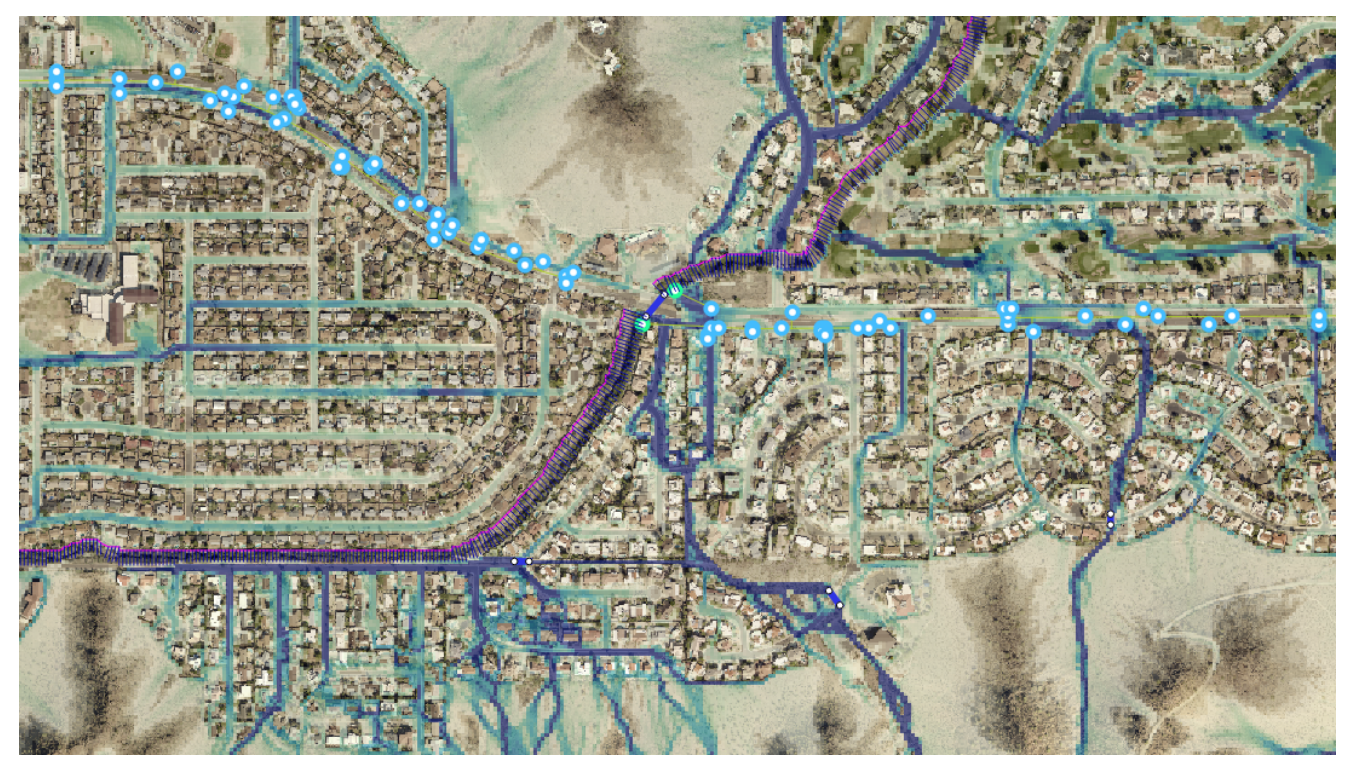

Figure 12: Shallow Urban Flooding Predicted FLO-2D

\section{Conclusions}

The QGIS plug-in tool for FLO-2D represents a significant advancement in cost effective development of complex urban flood models. A significant portion of the FLO-2D data input is based on interpolated shapefiles and raster data that might include thousands of buildings and walls, spatially variable rainfall and infiltration, and extensive storm drain data. As computer resources enable the more efficient assimilation of geographical and topographical mega data, modelers are simulating floods with smaller grid elements to generate higher flood map resolution. FLO-2D has more urban flood detail and components than any other comparable flood model. Creating the FLO-2D QGIS plugin provides flood managers and engineers access to an affordable and easy-to-apply urban flood routing system.

\section{References}

FLO-2D. (2018). FLO-2D Storm Drain Manual. Nutrioso: FLO-2D Software Inc. O' Brien, J. S. (2012). FLO-2D Reference Manual. Nutrioso: FLO-2D Software Inc. QGIS. (2018). QGIS Users Guide. Open Source Geospatial Foundation Project. 